\title{
Towards Skin Tissue Oxygen Monitoring: An Investigation of Optimal Visible Spectral Range and Minimal Spectral Resolution
}

\author{
Audrey Huong ${ }^{1, *}$, Kim Gaik Tay ${ }^{1}$, Xavier $\operatorname{Ngu}^{1,2}$ \\ ${ }^{1}$ Faculty of Electric and Electronic Engineering, Universiti Tun Hussein Onn Malaysia, Malaysia \\ ${ }^{2}$ Centre for Applied Electromagnetics, Universiti Tun Hussein Onn Malaysia, Malaysia
}

Received July 28, 2019; Revised October 5, 2019; Accepted December 15, 2019

Copyright $\bigcirc 2019$ by authors, all rights reserved. Authors agree that this article remains permanently open access under the terms of the Creative Commons Attribution License 4.0 International License

\begin{abstract}
This paper investigated the minimum spectral resolution and optimal spectra range for optical monitoring of skin blood oxygen saturation $\left(S_{t} O_{2}\right)$. For this purpose, this study recruited fourteen Asian volunteers for demonstration work. The performed measurements included reflectance information collected from the recruits during at rest and after a pressure of $140 \mathrm{mmHg}$ was applied on their upper right arm. The prediction of the required value was via analysis of data of the considered visible ranges of $500-660 \mathrm{~nm}, 520-645 \mathrm{~nm}, 520-600$ $\mathrm{nm}$, and $530-570 \mathrm{~nm}$, with sampling resolutions of $1 \mathrm{~nm}$, $2 \mathrm{~nm}, 5 \mathrm{~nm}$, and $10 \mathrm{~nm}$. The offline data analysis using Extended Modified Lambert Beer model revealed that the $\mathrm{S}_{t} \mathrm{O}_{2}$ value predicted using spectral data in the range 530 $570 \mathrm{~nm}$ showed considerable similarity with that obtained from $520-645 \mathrm{~nm}$ range at spectral resolutions of up to 5 $\mathrm{nm}$. The mean and standard deviation of the differences in the values obtained from these spectral ranges in the case of $5 \mathrm{~nm}$ sampling is given by $7 \pm 8.6 \%$ and $14 \pm 3.2 \%$ for at rest and blood occlusion experiment, respectively. The higher variability in the value predicted for the latter experiment is likely due to insufficiency of the employed technique to extricate the distinctive features in hemoglobin spectra using the corresponding resolution. The findings of this study may be useful as a guide to facilitate in the design of multispectral imaging system for optical monitoring of one's $\mathrm{S}_{t} \mathrm{O}_{2}$ with high speed.
\end{abstract}

Keywords Extended Modified Lambert Beer, Multispectral, Sampling Resolution, Skin Tissue Oxygen, Visible Spectral Range

\section{Introduction}

The investigation of $S_{t} O_{2}$ is of the utmost importance in the study of skin grafting, wound healing and skin rejuvenation to determine sustainability of living cells $[1,2]$. This is largely owing to the fact that oxygen is one of the important factors influencing the rate of cell growth and regulation of metabolism processes. Therefore information on $S_{t} O_{2}$ of the investigated skin site would allow one to evaluate the general health of the local cells and predict the healing outcomes of a wound, so that further effective intervention may be undertaken.

The measurement of $S_{t} O_{2}$ can be performed via either invasive or non-invasive means. While the invasive approach, which required drawing of a small sample of blood for measurement using arterial blood gas analyser, is the common practice in the clinical settings, the non-invasive technique is often the preferable option. The use of the latter is often based on the optical approach; it is able to provide a quick and painless experience to its users. Multispectral and hyperspectral imaging modalities are among the frequently employed optical spectroscopy technique in the study of physical and structural properties of the examined medium. Both systems produce a three-dimensional data containing spatial and spectral information of the investigated medium. Previous studies showed a remarkable performance of hyperspectral imaging technology in healthcare industries such as non-invasive assessment of superficial wound healing progress $[3,4]$ and in visualization of tumor progression [5]. These works analyzed reflectance data in the visible regions in their clinical diagnosis. There is, however, variation in the selection of wavelength range and spectral resolution used. Miclos et al. [6] used wavelength range of $500-660 \mathrm{~nm}$ at spectral resolution of $2 \mathrm{~nm}$ in the estimation of cutaneous tissue oxygen level, whereas other works showed percent of oxygen level in sickle cell disease patients [7] and in investigations of retinal oxygen levels [8] using $520-645 \mathrm{~nm}$ range. A similar work by 
Huong et al. [4] suggested the use of $520-600 \mathrm{~nm}$ at step size of $10 \mathrm{~nm}$ in the prediction of oxygen saturation for clinical assessment of acute wounds healing. This contributed to the variation in the $S_{t} O_{2}$ value reported in these studies; this variability was exacerbated with differences in the experimental conditions such as the optical arrangement, detector performance, and selected skin site [9].

Meanwhile many studies recently focused on removing redundant spectral bands than that is necessary for analysis, and considered only the distinct ones that have high sensitivity to the underlying physical properties. These were chosen using either supervised or unsupervised principle component analysis [10], or machine learning approach [11]. However, the selection was inconclusive. In general, the chosen spectral band should be a compromise between longer ranges placing higher demand on acquisition and processing time, and shorter ones providing insufficient information for the prediction of the required value. Besides a very fine sampling interval may not necessarily improve the accuracy of prediction, and a reasonably good result could be achieved at a course resolution. It is, therefore, the aim of this research to propose the most probable wavelength range and minimal spectral resolution suitably used for optical investigation of $S_{t} O_{2}$. The findings of this work would be useful in the design of multispectral system for optical monitoring of $S_{t} O_{2}$ with high sampling and fast computation time.

\section{Materials and Methods}

\subsection{Experiment System and Subject}

A diagram of the arrangement of the reflectance point spectroscopy system used in this study is drawn in Fig. 1. The illumination source used was a $9 \mathrm{~W}$ white light emitting diode (LED) (Model no. SMD 5730 from Aira Technologies) held in place using a make-shift stand. The illumination was at a distance of $90 \mathrm{~mm}$ from targeted site and an angle of $25^{\circ}$ from normal. This work used point spectroscopy device (USB 4000-UV-VIS from Ocean Optics Inc., Florida) for measurement of the reflected light intensity spectrum. The separation between the tip of the fiber optic probe and the investigated skin site was approximately $10 \mathrm{~mm}$ to allow a non-contact measurement of the reflectance signals. The detection was at an angle of approximately $15^{\circ}$ from normal. The experiment started with collection of reflectance data from white reference (spectralon panel from Labsphere, Inc.) and dark reference (with the tip of the fiber optic covered).

This work recruited fourteen $(n=14)$ Asian volunteers (seven males and seven females) aged between 22 and 26 years to participate in this study. This study was approved by the medical executive committee in the clinical center of Universiti Tun Hussein Onn Malaysia (UTHM). The subjects were the home students at UTHM. Each recruit self-declared a non-smoker, and has no history of hypertension and underlying illness; they gave their written consent prior to the measurement. These volunteers were instructed to rest in a chair for at least five minutes before the experiment. Next, they were required to place their right hand at a level close to their heart and against a black matte background shown in Fig. 1 before reflected signals were collected from their right thumb. These experiment procedures followed that described in [12]. These recruits were advised to remain still throughout the experiments conducted in a dark, quiet room. The detection integration time was chosen as $60 \mathrm{~s}$ while scanning to average and filtering function in SpectraSuite software were both turned off. In the arterial blood occlusion experiment oxygen carrying blood was blocked from flowing into the lower extremities. This is via inflation of an arm pressure to $140 \mathrm{mmHg}$ on upper right arm of the same subjects for two minutes. The same experiment procedure as described above was repeated immediately after the inflation time.

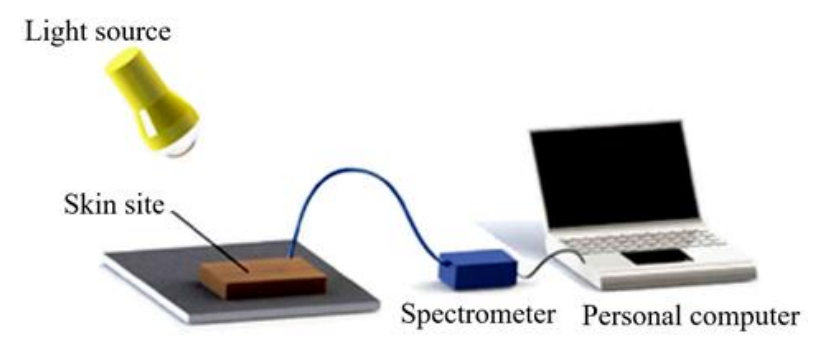

Figure 1. Optical arrangement of point spectroscopy system

The employed 16-bit spectrometer shown in Fig. 1 is able to detect and measure light intensity spectrum across wavelengths ranging from $178 \mathrm{~nm}$ to $893 \mathrm{~nm}$ at a spectral resolution of $0.2 \mathrm{~nm}$. However, we considered only spectral data in the visible region of $500-660 \mathrm{~nm}, 520-$ $645 \mathrm{~nm}, 520-600 \mathrm{~nm}$ and $530-570 \mathrm{~nm}$ owing to the large variability in the hemoglobin light absorption across these ranges. Light of visible range probed at a skin depth of $550-650 \mu \mathrm{m}$ [13], therefore the predicted $S_{t} O_{2}$ is presumably the mean of that of capillaries, arteries and veins located down to papillary dermis layer. Next, the acquired data were down sampled offline to different sampling rate of $1 \mathrm{~nm}, 2 \mathrm{~nm}, 5 \mathrm{~nm}$ and $10 \mathrm{~nm}$ for the investigation works. This work required the information of wavelength dependent light attenuation, $A(\lambda)$, calculated from (1) in the estimation of one's $S_{t} O_{2}$.

$$
A(\lambda)=\log \left(\frac{I_{W}(\lambda)-I_{D}(\lambda)}{I_{S}(\lambda)-I_{D}(\lambda)}\right)
$$

The symbols $I_{W}, I_{D}$ and $I_{S}$ represent reflectance data from white and dark reference, and from the targeted skin, respectively. 


\subsection{Analytic Model and Data Fitting Routine}

We considered Extended Modified Lambert Beer model (EMLB) previously proposed in Huong and Ngu [13] in the prediction of blood oxygen and carbon monoxide saturation. The light absorption, $\mu_{a}$, dependent light attenuation is given by:

$$
A(\lambda)=G_{0}+\mu_{a}(\lambda) d_{0}+G_{1}+\lambda e^{-\mu_{a}(\lambda) d_{1}} .
$$

This model proved to be an agreeable approximation of light attenuation as a function of light scattering and absorbing in an absorbing turbid medium. Equation (2) included the effects of light absorption by both melanin and hemoglobin. The representation and detailed description of each term can be found in [13]. Since the recruited volunteers were non-smokers, and declared that they did not recently expose themselves to any poisonous gases, it was assumed that the carboxyhemoglobin level in their blood is negligible. The wavelength dependent total light absorption as a function of $\mathrm{S}_{t} \mathrm{O}_{2}$ and extinction coefficient ( $\varepsilon$ ) of oxyhemoglobin, $\mathrm{HbO}_{2}$, and deoxyhemoglobin, $\mathrm{Hb}$, is given by [4]:

$$
\mu_{a}(\lambda)=\left(\left(\varepsilon_{H b O 2}(\lambda)-\varepsilon_{H b}(\lambda)\right) S_{t} O_{2}+\varepsilon_{H b}(\lambda)\right) T .
$$

The value of $\varepsilon_{H b O 2}$ and $\varepsilon_{H b}$ can be found in [14] while the value of total hemoglobin concentration, $T$, is taken here as $268 \mu \mathrm{M}$. Substituting (3) into (2) gives an expression of light attenuation as a function of $S_{t} O_{2}$. The optimization fitting function used in this work was based on fminsearch in MATLAB to non-linearly search for the optimum value for all the unknowns $\left(G_{0}, d_{0}, G_{l}, d_{l}\right.$ and $S_{t} O_{2}$ ) in (2). This fitting routine did not apply boundary to the value of each unknown. The fitting iteration was set to 200 , and the search of the optimized value was based on the calculated difference between the measured light attenuation in (1) and the value given from (2), $\Delta A$. This fitting process was terminated when either the iteration limit has exceeded or the sum of $\Delta A^{2}$ is lesser or equal to $10^{-10}$.

\section{Results and Analysis}

The $S_{t} O_{2}$ predicted for each recruited volunteer during at rest and arterial blood occlusion experiment using data with spectral resolution of $1 \mathrm{~nm}, 2 \mathrm{~nm}, 5 \mathrm{~nm}$ and $10 \mathrm{~nm}$ across the considered four wavelength ranges is shown on the left panels of Fig. 2. Shown on the right panels of Fig. 2 is the overall mean and standard deviation in the $S_{t} O_{2}$ predicted using data of different wavelength ranges. The results revealed high repeatability in the $S_{t} O_{2}$ calculated as $87.9 \pm 4.2 \%$ estimated for at rest condition throughout different wavelength ranges using sampling step of no greater than $5 \mathrm{~nm}$ in Fig. 2(a) - (c). Meanwhile a lower mean with larger variability of $36.16 \pm 18 \%$ is observed in the case of blood occlusion.

Since spectral data with sample interval of $1 \mathrm{~nm}$ has the highest information content, the predictions shown in Fig. 2(a) are used here as the gold standard. In addition it was also presumed that analysis using highly sampled data is likely to be unaffected by the presence of noise in the signals. The differences in the prediction using that between interval $1 \mathrm{~nm}$ (Fig. 2 (a)) and other lower spectral resolutions shown in Fig. 2(b)-(d) were statistically analyzed using a two-tailed independent samples $t$-test analysis with confidence level of $95 \%$ (IBM SPSS statistic 22 ). While the majority of the results showed statistical significance with $\rho>0.05$ for $S_{t} O_{2}$ estimated for the conducted experiments, $\rho<0.05$ was calculated for $S_{t} O_{2}$ from at rest and blood occlusion experiment using sampling step of $10 \mathrm{~nm}$ across wavelength range of $520-$ $600 \mathrm{~nm}$ and $530-570 \mathrm{~nm}$, respectively.
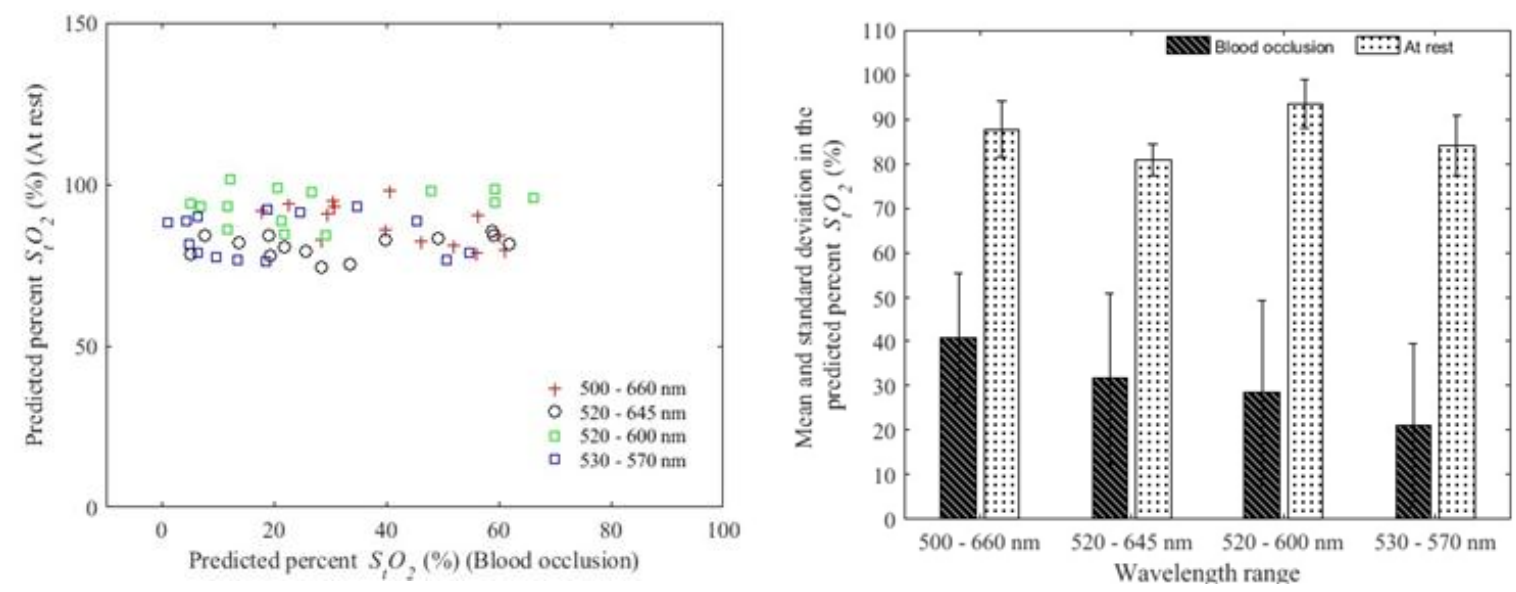

(a) Spectral resolution: $1 \mathrm{~nm}$ 

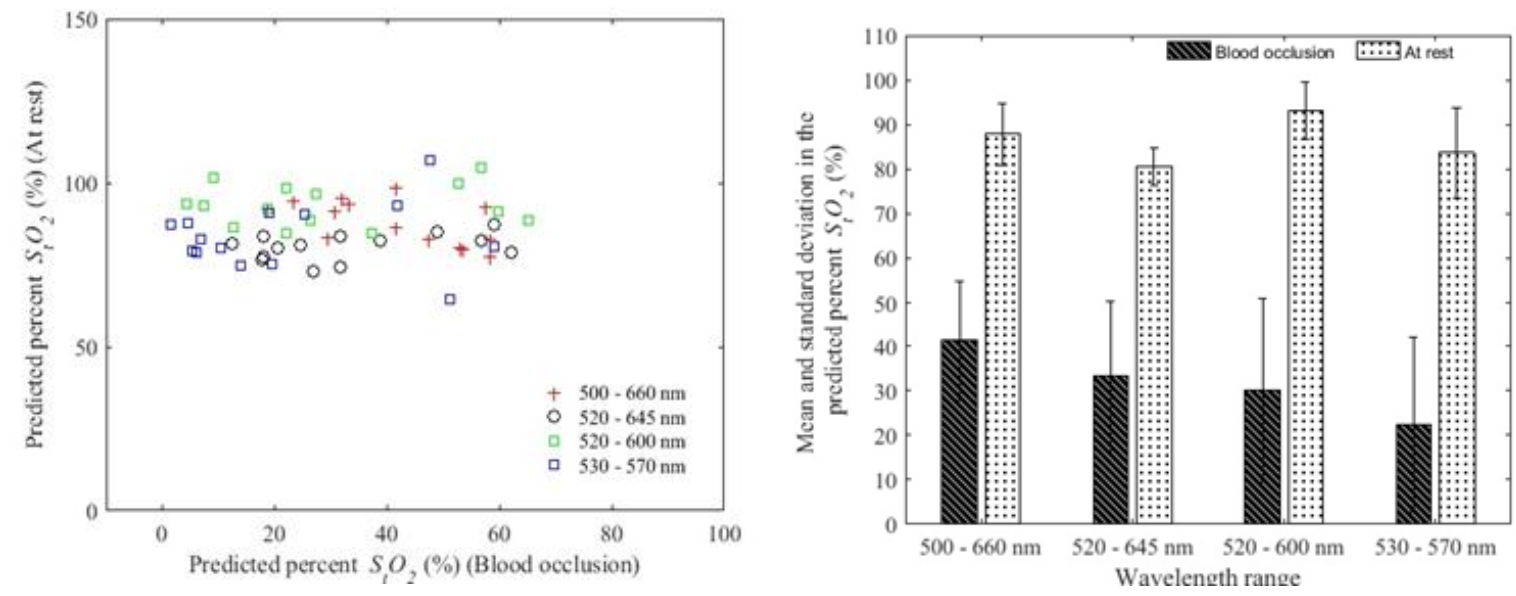

(b) Spectral resolution: $2 \mathrm{~nm}$
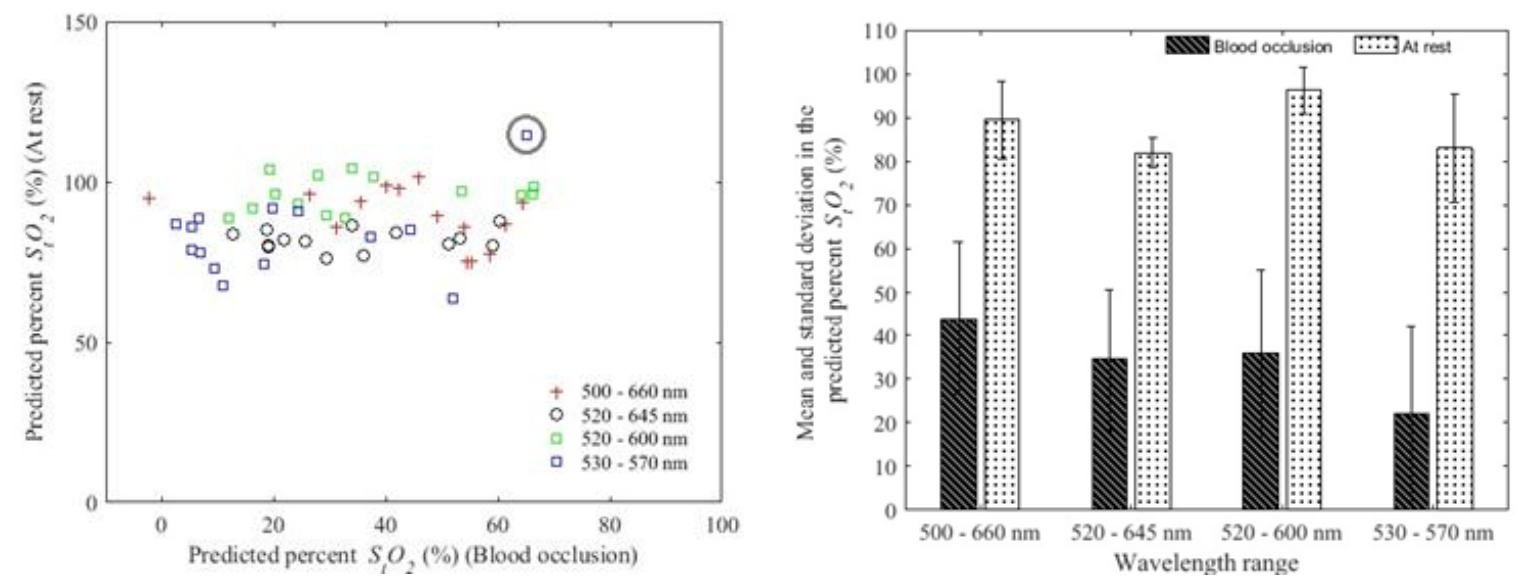

(c)Spectral resolution: $5 \mathrm{~nm}$
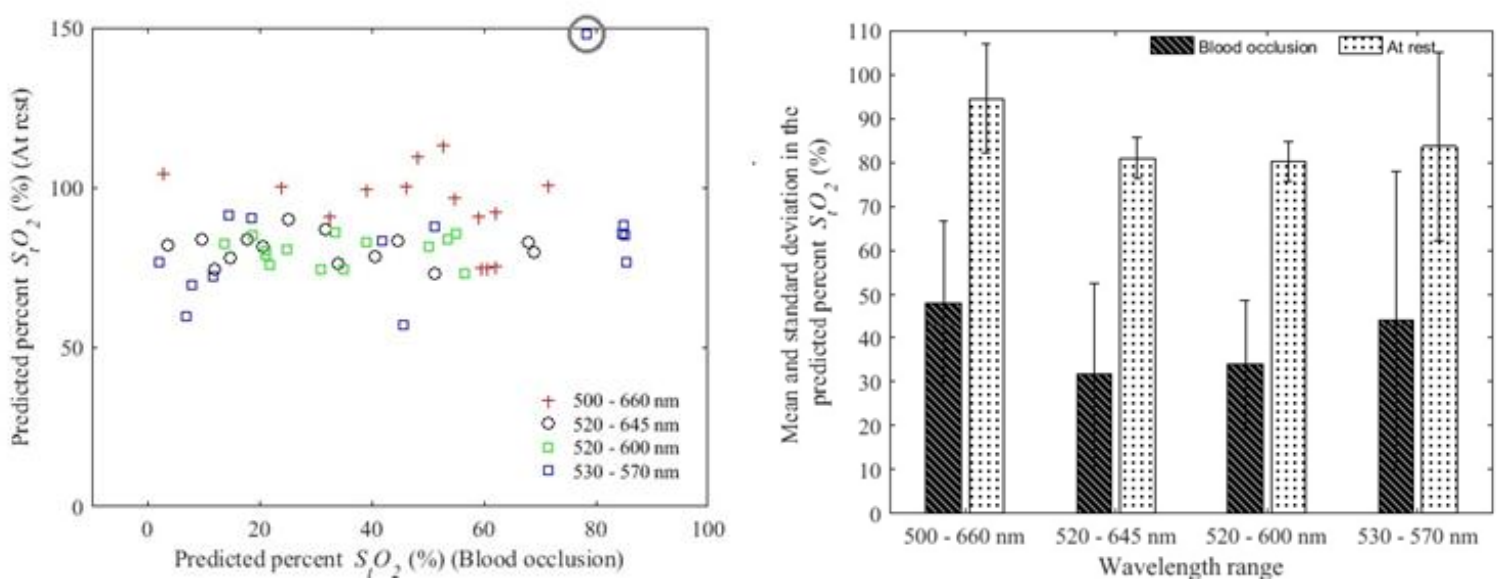

(d)Spectral resolution: $5 \mathrm{~nm}$

Figure 2. (Left) The $S_{t} O_{2}$ predicted for volunteers $(n=14)$ during at rest and arterial blood occlusion condition using spectral data of different wavelength range and sampling interval a - d. (Right) The mean and standard deviation (error bar) in the $S_{t} O_{2}$ predicted using data of different spectral range 


\section{Discussion}

Based on the results shown in Fig. 2, it is shown that different wavelength range yielded differences in the approximated $\mathrm{S}_{t} \mathrm{O}_{2}$. The results revealed that the predicted mean values did not show a gradual trend with either an increase in the wavelength range or sampling interval. A relatively high consistency in the $\mathrm{S}_{t} \mathrm{O}_{2}$ value of $80-90 \%$ for at rest condition is obtained using wavelength range of $530-570 \mathrm{~nm}$ and its longer range counterparts, and across different sampling intervals. All the considered wavelength ranges showed a decrease in the calculated $\mathrm{S}_{t} \mathrm{O}_{2}$ following the disruption of oxygen supply to the local tissues. The greatest change in the predicted value with interruption of tissue microcirculation is via the use of 520-600 nm range. There is, however, significant inconsistency between its predictions using $10 \mathrm{~nm}$ and $1 \mathrm{~nm}$ sampling interval $(\rho=$ $0.000)$ during at rest condition.

The 520-645 $\mathrm{nm}$ range shows a striking consistency in the value estimated using different sample intervals and with varying experiment conditions shown in Fig. 2. This is followed by the value given by the $530-570 \mathrm{~nm}$ range whose predictions were considerably similar to that produced using 520 - 645 $\mathrm{nm}$ range for sampling size of up to $5 \mathrm{~nm}$. A comparison in the value given by both of these ranges using $5 \mathrm{~nm}$ sampling step shows an absolute mean difference of $7 \pm 8.6 \%$ and $14 \pm 3.2 \%$, respectively, for the $\mathrm{S}_{t} \mathrm{O}_{2}$ predicted for at rest and occlusion experiment. The greatest difference is, however, observed for $\mathrm{S}_{t} \mathrm{O}_{2}$ during occlusion experiment in Fig. 2(d) using this smallest wavelength range and with the largest sampling step (i.e. $10 \mathrm{~nm})$. This is supported by the computed $\rho$ value of 0.03 in the statistical test between the results from sampling interval of $10 \mathrm{~nm}$ and $1 \mathrm{~nm}$ for blood occlusion experiment. It must be mentioned that the use of long wavelength range (i.e. $500-660 \mathrm{~nm}$ ) does not necessarily guarantee consistency in the results with different sampling size; thus suggesting the possibility of data oversampling using this range than that is necessary in the estimation of the required value.

The results in Fig. 2 show that the variability is larger for blood occlusion measurement than that is for at rest condition. This may be explained by the change in the optical signature of hemoglobin under these conditions. The presence of troughs and peaks in the light attenuation spectrum detected under high $\mathrm{S}_{t} \mathrm{O}_{2}$ condition (at rest condition) can clearly be seen in the visible range as can be illustrated in [14]; they are contributed largely by the absorptivity of oxyhemoglobin. This variability is gradually reduced to a single hump following a decrease in the $\mathrm{S}_{t} \mathrm{O}_{2}$ value (blood occlusion condition); thus suggesting the dependence of the employed analytic technique on the distinct bands that are sensitive to the underlying medium absorption.

Figure 2 shows an increase in the variability in the value with increasing sampling size. Since the employed analytical technique used fitting approach in its prediction, a lack in the sample size is likely to be more sensitive to noise in the signals hence affecting the overall performance. This might be the reason for the presence of outliers marked by circle observed for the same recruit when using larger sampling steps (i.e. $5 \mathrm{~nm}$ and $10 \mathrm{~nm}$ ). In addition, the variability in the $\mathrm{S}_{t} \mathrm{O}_{2}$ can also be contributed by differences in the physical and environmental factors such as daily activity, lifestyle, skin condition, diets and health status of each individual.

Even though there is no commonly accepted gold standard to the measurement of $S_{t} O_{2}$ as this value may vary depending on the experimental conditions and the investigated skin regions, an overall result shows that all the considered wavelength ranges are viable in the monitoring of oxygen saturation. In this regard, a relative measurement of $\mathrm{S}_{t} \mathrm{O}_{2}$ would be more suitable to evaluate and compare the tissue oxygenation status following medical treatment or intervention. Thus far, the 520-645 range emerged as the optimum range with the highest robustness to noise and there is high repeatability in the value predicted using different spectra resolutions. Nonetheless even with the $10 \mathrm{~nm}$ sampling step, the use of this range in the design of multispectral imaging system would place a high demand on the system required for data acquisition, transportation, processing and storage. This is as compared to $530-570 \mathrm{~nm}$ range with sampling resolution of $5 \mathrm{~nm}$ (that produced nine bands), which produced predictions similar to that from its $520-645 \mathrm{~nm}$ counterpart.

\section{Conclusions}

This study considered a variance in the wavelength range and data sampling interval in the prediction of $\mathrm{S}_{t} \mathrm{O}_{2}$. This work observed a notable similarity in the value estimated using wavelength range of $520-645 \mathrm{~nm}$ and $530-570 \mathrm{~nm}$ for different experiment conditions using sampling resolutions of up to $5 \mathrm{~nm}$. This study attributed inconsistency in the predicted value following a disruption in the tissues oxygen supply to the lack in the optical sensitivity of the chosen wavelength bands. This scenario is aggravated under the undersampling setting when the analytic approach may be more sensitive to the noise presence in the signals. This study concluded that the use of spectral data of wavelength range of $530-570 \mathrm{~nm}$ and sampling size of $5 \mathrm{~nm}$ may have potential for using in the design of multispectral imaging system with high speed.

\section{Acknowledgements}

We would like express our gratitude to Ministry of Education in Malaysia (K038, 1581) for financially supporting this study. Special thanks also go to all 
volunteers participated in the experiments.

\section{REFERENCES}

[1] S. Dixit, D. R. Baganizi, R. Sahu, E. Dosunmu, A. Chaudhari, K. Vig, S. R. Pillai, S. R. Singh, V. A. Dennis. Immunological challenges associated with artificial skin grafts: available solutions and stem cells in future design of synthetic skin, Journal of Biological Engineering, Vol. 11, No. 49 2- 23, 2017.

[2] H. F. Jelinek, D. J. Cornforth, K. Blackmore. Visualisation in biomedicine as a means of data evaluation, Journal of Visualization, Vol. 14, No. 4, 353- 359, 2011.

[3] R. Rutkowski, M. Schuster, J. Unger, C. Seebauer, H. R. Metelmann, Th. V. Woedtke, K. D.Weltmann, G. Daeschlein. Hyperspectral imaging for in vivo monitoring of cold atmospheric plasma effects on microcirculation in treatment of head and neck cancer and wound healing, Clinical Plasma Medicine, Vol. 7, 52-57, 2017.

[4] A. Huong, S. Philimon, X. Ngu. Multispectral imaging of acute wound tissue oxygenation, Journal of Innovative Optical Health Sciences, Vol. 10, No. 3, 1750004- 10, 2017.

[5] D. R. McCormack, A. J. Walsh, W. Sit, C. L. Arteaga, J. Chen, R. S. Cook, M. C. Skala. In vivo hyperspectral imaging of microvessel response to trastuzumab treatment in breast cancer xenografts, Biomedical Optic Express, Vol. 5, No. 7, 2247-2261, 2014.

[6] S. Miclos, S. V. Parasca, M. A. Calin, D. Savastru, D. Manea. Algorithm for mapping cutaneous tissue oxygen concentration using hyperspectral imaging, Biomedical Optic Express, Vol. 6, No. 9, 3420-3430, 2015.

[7] K. J. Zuzak, M. T. Gladwin, R. O. Cannon III, I. W. Levin. Imaging hemoglobin oxygen saturation in sickle cell disease patients using noninvasive visible reflectance hyperspectral techniques: effects of nitric oxide, American Journal of Physiology Heart and Circulatory Physiology, Vol. 285, No. 3, 1183-1189, 2003.

[8] E. O. Olweny, S. Faddegon, S. L. Best, N. Jackson, E. F. Wehner, Y. K. Tan, K. J. Zuzak, J. A. Cadeddu. Renal oxygenation during robot-assisted laparoscopic partial nephrectomy: characterization using laparoscopic digital light processing hyperspectral imaging, Journal of Endourology, Vol. 27, No. 3, 265-269, 2013.

[9] S. P. Philimon, A. K. C. Huong, X. T. I. Ngu. Investigation of spatial resolution dependent variability in transcutaneous oxygen saturation using point spectroscopy system, IOP Conference Series: Materials Science and Engineering, Vol. 226, 1-8, 2017.

[10] K. Sun, X. Geng, L. Ji, Y. Lu. A New Band Selection Method for Hyperspectral Image Based on Data Quality, IEEE Journal of Selected Topics in Applied Earth Observations and Remote Sensing, Vol. 7, No. 6, 2697-2703, 2014.

[11] X. Zhou, S. Li, F. Tang, K. Qin, S. Hu, S. Liu. Deep Learning With Grouped Features for Spatial Spectral Classification of Hyperspectral Images, IEEE Geoscience and Remote Sensing Letters, Vol. 14, No. 1, 97-101, 2017.

[12] I. V. Meglinski, S. J. Matcher. Modelling the sampling volume for skin blood oxygenation measurements, Medical and Biological Engineering and Computing, Vol. 39, No. 1, 44- 50, 2001.

[13] A. Huong, X. Ngu. The application of extended modified Lambert Beer model for measurement of blood carboxyhemoglobin and oxyhemoglobin saturation, Journal of Innovative Optical Health Sciences, Vol. 7, No. 3, 1450026-33, 2014.

[14] W. G. Zijlstra, A. Buursma, O. W. van-Assendelft. Visible and near infrared absorption spectra of human and animal haemoglobin: determination and application, VSP, Netherlands, 2000. 